\title{
Background gravity correction to the limiting mass of white dwarfs
}

\author{
Anarya Ray ${ }^{1, a}$, Pronobesh Maity ${ }^{2, b}$, Parthasarathi Majumdar ${ }^{3, c}$ \\ ${ }^{1}$ Department of Physics, Presidency University, Kolkata 700073, India \\ ${ }^{2}$ International Centre for Theoretical Sciences, Bengaluru 560089, India \\ ${ }^{3}$ Department of Physics, Ramakrishna Mission Vivekananda Educational and Research Institute, Belur 711202, West Bengal, India
}

Received: 23 January 2018 / Accepted: 20 January 2019 / Published online: 31 January 2019

(C) The Author(s) 2019

\begin{abstract}
While computing the Fermi degeneracy pressure of electrons in a white dwarf star within the framework of hydrostatic equilibrium, we depart from the extant practice of treating the electrons as a free fermion gas, by including the effect of the background gravitational potential experienced by the electrons in the star, resulting from the mass of its constituent atoms (being the mass of all nucleons). Modifying the free particle Hamiltonian with this effective potential, we employ first order quantum mechanical perturbation theory to compute the degeneracy pressure, in order to study the effect of inclusion of this self-gravity of the star on the limiting mass. The final effect is found to be non-trivial, but perhaps a shade too small to alter any major observational result.
\end{abstract}

\section{Introduction}

White Dwarfs have been a phenomenon of great importance to astrophysicists, as also theoretical physicists since their discovery in 1910. A White Dwarf is a stellar remnant, the fate of certain stars after they have exhausted all of their nuclear fuel. They are extremely dense with masses comparable to that of the Sun but a volume comparable to that of the earth. The issue of a limiting mass for white dwarf stars has been an active area of research since the 1930s till date. General Relativity has been applied recently $[1,2]$ to realize and augment the hydrostatic equilibrium scenario of the incipient works [3] on the subject, leading to fractional changes on the maximum mass of white dwarfs. The incorporation of large magnetic fields has also been examined [4-6], leading to an apparent enhancement in the limiting mass-yielding the so-called super-Chandrasekhar white dwarfs. The inclusion of rotation and finite temperatures [7] have also been made recently in order to estimate their effect on the limiting mass.

\footnotetext{
a e-mail: ronanarya9988@gmail.com

b e-mail: pronobesh.maity@icts.res.in

c e-mail: bhpartha@gmail.com
}

In the early derivations of the limiting mass of a white dwarf star using hydrostatic equilibrium by Anderson [8], Stoner [9] and Landau [10], and Chandrasekhar [11,12], due to relativistic effects necessitated by the density of matter of these white dwarfs, the assumption has been made that the electrons inside the star can be modelled as free quantum mechanical particles inside a spherical well with the radius of the star. The electrons are of course assumed to be trapped in an infinite well, since, to escape outside from the boundary, the electrons would need to overcome a huge Coulombic potential. Such a potential is clearly absent inside, as the star as a whole is electrically neutral. But inside the well, unlike the electrostatic interaction, gravity does not cancel out. The electrons inside the well are not free but are actually residing in a background gravitational potential. Since this background potential is admittedly weak, the electrons can be more realistically modelled approximately as particles inside an infinite spherical well, but with an additional perturbation by this background gravitational potential. The aim of this article is to explore the effects of such a background potential on the mass limit. We argue that a correction to the limiting mass, albeit a small one, does indeed emerge within our proposed modification of electron dynamics inside the star. We also note that this is a new physical effect that has not been considered heretofore, despite the large literature on the subject.

In some of the very early work on white dwarf limiting mass (see Refs. [8-10]), the hydrostatic equilibrium has been realized, not as a balance of gravitational pressure and Fermi degeneracy pressure of relativistic electrons, but by a minimization of the total energy of the star. The equivalence, of the energy minimization approach of Anderson, Stoner and Landau to the pressure balance viewpoint endorsed by Chandrasekhar, goes to the extent of yielding the correct Equation of State (EoS) for the electrons, as can be shown by computing the derivative of the kinetic energy of the star with respect to its volume: for the non-relativistic case, this yields 
the $\operatorname{EoS} P \propto \rho^{5 / 3}$; for the relativistic case of Chandrasekhar and his precursors one obtains $P \propto \rho^{4 / 3}$.

A perturbative correction to the relativistic equation of state, due to the background gravitational potential proposed by us, is therefore a new result of this paper, which has never appeared in the white dwarf literature. Of course, in this paper very important effects such as those due to magnetic fields and rotation as well as those due to general relativistic corrections, are ignored. These have been discussed in detail in Refs. [1-7]. Our aim here, instead, is to capture the essence of the new physics associated with the electron degeneracy in presence of the gravitational potential of the atoms of the star, in as simple a context as possible, leaving other complexities aside. In doing so, we have gone back to the incipient treatments based on Newtonian gravity, because this is adequate to ascertain how big the correction is, in order of magnitude, to the limiting mass. In this same spirit, we also adopt the rather drastically approximate model of a uniform spherical star, rather than follow Chandrasekhar's inclusion of non-uniformity of density as a more realistic feature of polytropic stars. Incorporation of these additional subtleties makes sense only when the correction we are after is large enough to justify a rigorous treatment.

The overview of the rest of the paper is as follows: we begin the next section with introducing the proposed modification due to the background gravity arising from the nuclei constituting the star, to the free electron Hamiltonian, within a sort of mean field picture. This modification is seen to be a weak effective gravitatonal potential. Resorting to first order time-independent perturbation theory, the effect of this effecive potential on the limiting mass of the star is estimated. We conclude with a few remarks on the viability of the various approximations made in the body of the paper.

\section{Proposed correction}

While calculating the electrons' average kinetic energy, Chandrasekhar assumed that the electrons are trapped in an infinite well inside which they are free. But in reality, they are not. The average Coulombic interaction may cancel out due to the fact that the star as a whole is electrically neutral. But an effective gravitational potential exists inside the spherical well which, despite being weak, can alter the quantum mechanical properties of the electrons and hence their zero point energy. We show that the order of magnitude correction to the limiting mass is in fact computable.

An electron inside a star at a distance $r$ from the radius experiences a gravitational field $(F)$ only due to the matter contained in a Gaussian sphere of radius $r$. Assuming uniform density for simplicity, the gravitational flux across the surface of the star is proportional to the stellar mass $M=(4 / 3) \pi r^{3} \rho$,

$$
\oint_{S} \vec{F} \cdot d \vec{S}=-4 \pi G \frac{4}{3} \pi r^{3} \rho
$$

so that, the gravitational force at every point inside the star can be written as

$\vec{F}(\vec{r})=-\frac{4 \pi G \rho \vec{r}}{3}$ for $r \leq a$,

while, for locations outside the star,

$\vec{F}(\vec{r})=-\frac{4 \pi G \rho a^{3}}{3 r^{2}} \frac{\vec{r}}{r}$ for $r>a$.

This leads to an effective self-gravitational potential affecting the elecron gas,

$V(\vec{r})=-\int_{\infty}^{r} F\left(\overrightarrow{r^{\prime}}\right) \cdot d \overrightarrow{r^{\prime}}$

The potential energy $U(\vec{r})$ is just $V(\vec{r})$ times the mass $m_{e}$ of the electron: $U(r)=\left(2 \pi m_{e} G \rho r^{2} / 3\right)-2 \pi G \rho m_{e} a^{2}$. Thus, in this scenario, the electrons are trapped in an infinite spherical well potential, with a weak harmonic potential inside the star. One can treat this weak background gravity potential as a perturbation on the unperturbed free electron dynamics for the infinite spherical well inside the star.

\subsection{Unperturbed star}

Recall that the infinite spherical well is given by

$$
\begin{aligned}
V(r) & =0 \text { for } r \leq a \\
& =\infty \text { for } r>a .
\end{aligned}
$$

Inside this well, the wavefunction has the structure appropriate to spherical symmetry: $\Psi_{n l m}(r, \theta, \phi)=R_{n l}(r) Y_{l m}(\theta, \phi)$.

\subsubsection{Non-relativistic wavefunction}

Defining the reduced wave function $u_{n l} \equiv r R_{n l}$, the radial Schrödinger equation assumes the form

$\frac{d^{2} u_{n l}}{d r^{2}}=\left[\frac{l(l+1)}{r^{2}}-k^{2}\right] u_{n l}$,

where $k^{2} \equiv 2 m_{e} E / \hbar^{2}$. Restricting our attention to $l=0$ states for simplicity, ${ }^{1}$ we observe that Eq. (6) has the solution

\footnotetext{
1 The reason for this simplifying assumption is our primary aim to determine an order-of-magnitude correction to the limiting mass, due to background gravity, through minimizing the energy with respect to $n$. The minimum value of $n$ being $n \equiv n_{0} \simeq 10^{8}$, such high values of $n$, and the fact that the perturbing potential is spherically symmetric imply together that $l \neq 0$ (with $l=O(1)$ ) states are unlikely to affect the first order perturbative correction to the energy eigenvalue estimate. Note that the incipient papers [8-12] exclusively consider the energy eigenvalues and/or the density of states corresponding to $l=0$ states, (which is obvious from their use of the energy spectrum corresponding
} 
$u_{n 0}(r)=A \sin k r+B \cos k r$.

Regularity of the radial wavefunction $R_{n 0}(r)$ at the origin $r=0$ implies that $B=0$. The boundary condition at the surface of the star $r=a$ implies that the wavefunction must vanish at this surface, which leads to the standard spectrum of the unperturbed system

$E_{n 0}=\frac{n^{2} \pi^{2} \hbar^{2}}{2 m_{e} a^{2}}, n=0,1,2, \ldots$

The correspondng unperturbed energy eigenstate is given by the normalized wavefunction,

$\Psi_{n 00}=\frac{1}{\sqrt{2 \pi a}} \frac{\sin (n \pi r / a)}{r}$.

\subsubsection{Relativistic (Dirac) wavefunction}

From Eq. (30) of [15], considering only the case of $l=0$ as before, using the boundary condition of vanishing largecomponent, which implies $k=\frac{n \pi}{a}$ and using $\frac{\hbar c k}{E+m_{e} c^{2}}=$

$$
\begin{aligned}
& \frac{\hbar c k}{\sqrt{\hbar^{2} c^{2} k^{2}+m_{e}^{2} c^{4}}+m_{e} c^{2}}=\frac{k}{\sqrt{k^{2}+\frac{m_{e}^{2} c^{2}}{\hbar^{2}}+\frac{m_{e} c}{\hbar}} \text { we get: }} \\
& \Psi_{n, 0, j=1 / 2, m}(r, \theta, \phi) \\
& =\mathcal{A}\left(\begin{array}{c}
j_{0}\left(\frac{n \pi r}{a}\right) Y_{1 / 2,0, m}(\theta, \phi) \\
\left.-\frac{i k}{\sqrt{k^{2}+\frac{1}{\lambda_{e}^{2}}}+\frac{1}{\lambda_{e}}} j_{1}\left(\frac{n \pi r}{a}\right) Y_{1 / 2,1, m}(\theta, \phi)\right) \\
\sqrt{E^{2}-m_{e}^{2} c^{4}}=\frac{n \pi \hbar c}{a}
\end{array}\right.
\end{aligned}
$$

where, $\mathcal{A}$ is the normalization constant, $Y_{j l m}(\theta, \phi)$ are the two-component normalized spherical harmonics, $j_{l}$ the spherical Bessel functions of the first kind and for mathematical convenience, we have defined $\frac{1}{\lambda_{e}}=\frac{m_{e} c}{\hbar}, m_{e}$ being the mass of the electron. Normalizing Eq. (10), we get:

$$
\begin{aligned}
\int \Psi^{\dagger} \gamma_{0} \Psi d^{3} r & =1 \\
\text { where, } \gamma_{0} & =\left(\begin{array}{cc}
\mathbf{I} & \mathbf{0} \\
\mathbf{0} & -\mathbf{I}
\end{array}\right) \\
\Longrightarrow \mathcal{A} & =\frac{\sqrt{\pi}|n|}{2 \sqrt{\frac{a^{3}}{\lambda_{e}}}}
\end{aligned}
$$

\footnotetext{
Footnote 1 continued
}

to the momentum spectrum $p=\frac{n \pi \hbar}{a}$ in place of $p=\frac{\zeta_{n l} \pi \hbar}{a}$ where $\zeta_{n l}$ is the nth zero of the lth spherical bessel function of the first kind) for the purpose of estimating the limiting mass. Since our focus is on the first order corrections to the limiting mass due to background gravity, we have followed the same practice of restricting to $l=0$ states. For a slightly longer discussion on this, section 3 of the paper may be perused.

\subsection{Background gravity correction}

\subsubsection{Non-relativistic approximation}

The first order perturbation correction to the energy spectrum, due to the background gravitational potential experienced by the electrons in the star, can be easily computed:

$$
\begin{aligned}
E_{n}^{1} & =\int d^{3} r \Psi_{n}^{*} U(r) \Psi_{n} \\
& =\frac{4 \pi G \rho}{3}\left(-\frac{4}{3} m_{e} a^{2}-\frac{m_{e} a^{2}}{4 n^{2} \pi^{2}}\right) .
\end{aligned}
$$

But

$\rho=\frac{M}{\frac{4}{3} \pi a^{3}}$.

Hence

$E_{n}^{1}=\frac{G M m_{e}}{a}\left(-\frac{4}{3}-\frac{1}{4 \pi^{2} n^{2}}\right)=\frac{\omega}{a}\left(-\frac{4}{3}-\frac{1}{4 \pi^{2} n^{2}}\right)$

where

$\omega=G m_{e} M$

The heavier the star is, the smaller and hence denser it is. The Fermi momentum, the threshold momentum of the electrons is an increasing function of density:

$p_{f}=\frac{\pi \hbar n_{f}}{a}=\left(\frac{4 \pi^{3} \hbar^{3} \rho}{A_{0} m_{p}}\right)^{1 / 3}$

where, $A_{0}$ is the average mass number of the nuclei and $m_{p}$ is the mass of the proton. For the average white dwarf, $\rho \simeq 10^{6} \mathrm{~g} / \mathrm{cc}$. This corresponds to momentum of the order of $\sim m_{e} c$. At such high momenta the electrons have to be treated relativistically. So, in ultra-relativistic approximation, kinetic energy with the above first-order perturbation is:

$$
\begin{aligned}
E(n)= & \frac{n \pi \hbar c}{a}+E_{n}^{1}=\frac{n \pi \hbar c}{a} \\
& +\frac{\omega}{a}\left(-\frac{4}{3}-\frac{1}{4 \pi^{2} n^{2}}\right) \equiv A n-B-\frac{D}{n^{2}}
\end{aligned}
$$

where

$A \equiv \frac{\pi \hbar c}{a}, B \equiv \frac{4 \omega}{3 a}, D \equiv \frac{\omega}{4 a \pi^{2}}$.

The numerical order of magnitude values of $A, B$ and $D$ have been calculated from the observed density and radius of typical white dwarf stars: $\rho \approx 10^{6} \mathrm{~g} / \mathrm{cm}^{3}, \mathrm{a} \approx 7000 \mathrm{~km}$ yielding $\mathrm{A} \approx 10^{-32} \mathrm{~J}, \mathrm{~B} \approx 10^{-23} \mathrm{~J}, \mathrm{D} \approx 10^{-25} \mathrm{~J}$. Here, the relatively large values of the constants $B$ and $D$, compared to that of the unperturbed constant $A$ raise a question of validity of the perturbative result. One expects that the perturbative result would not dominate the zeroth order unperturbed result 
corresponding to the original scenario of the free electron gas; thus

$E(n) \gg 0 \Rightarrow A n \gg B+\frac{D}{n^{2}}$.

Since the correction terms decrease with increasing $n$, there must be a minimum $n=n_{0}$ such that $E\left(n_{0}\right)=0$; any value of $n \gg n_{0}$ is acceptable as a perturbative correction.

To find $n_{0}$, note that one has to solve a cubic equation; this can be done exactly, without any assumption on $D$, yielding $n_{0} \approx 10^{9}$. This implies that our perturbative correction is valid only for electron states lying within the domain $n_{0} \ll n<n_{f}$, and this is consistent with our analysis in the ultrarelativistic limit. The density of states is, of course, still given by $g(n) d n=\pi\left(n^{2} / 2\right) d n$.

The total kinetic energy of the electrons can be computed using the formula

$E_{k i n}=\int_{0}^{n_{f}} 2 E_{n} g(n) d n$,

with only the $n=0$ lower limit of the integration being replaced by $n_{0}$; this gives us the result

$$
\begin{aligned}
E_{k i n}= & \int_{n_{0}}^{n_{f}}\left(A n-B-\frac{D}{n^{2}}\right) \pi n^{2} d n \\
= & \pi A \frac{n_{f}^{4}}{4}\left[1-\left(\frac{n_{0}}{n_{f}}\right)^{4}\right]-\pi B \frac{n_{f}^{3}}{3}\left[1-\left(\frac{n_{0}}{n_{f}}\right)^{3}\right] \\
& -\pi D n_{f}\left(1-\frac{n_{0}}{n_{f}}\right) .
\end{aligned}
$$

Now, $n_{f} \approx N^{\frac{1}{3}} \approx 10^{20}$, since, $N \approx 10^{60}$, it follows that $\frac{n_{0}}{n_{f}} \approx 10^{-11}$, so that all powers of $n_{0} / n_{f}$ can be safely ignored.

With these approximations, and substituting the expressions for the constants $A, B, D$, the total kinetic energy can be written as

$$
\begin{aligned}
E_{k i n}= & C_{1} \frac{\hbar c}{a}\left(\frac{Z}{A_{0} m_{p}}\right)^{\frac{4}{3}} M^{\frac{4}{3}}-C_{2} \frac{G m_{e}}{a}\left(\frac{Z}{A_{0} m_{p}}\right)^{\frac{1}{3}} M^{\frac{4}{3}} \\
& -C_{3} \frac{G m_{e}}{a}\left(\frac{Z}{A_{0} m_{p}}\right) M^{2}
\end{aligned}
$$

where, $C_{1}=(243 / 256)^{1 / 3} \pi, C_{2}=\left(\frac{3}{\pi}\right)^{\frac{1}{3}} \frac{1}{4 \pi}, C_{3}=$ $\frac{4}{3}, A_{0} m_{p}$ is an average nuclear mass, $a$ is the radius of the star, $M$ is the mass of the star.

The total energy with the perturbative correction can now be written as

$$
\begin{aligned}
E_{t o t}= & E_{k i n}-3 G \frac{M^{2}}{5 a} \\
= & \frac{M^{\frac{4}{3}}}{a}\left(C_{1} \frac{h c Z^{\frac{4}{3}}}{\left(A_{0} m_{p}\right)^{\frac{4}{3}}}-C_{2} \frac{G m_{e} Z^{\frac{1}{3}}}{\left(A_{0} m_{p}\right)^{\frac{1}{3}}}\right) \\
& -\frac{M^{2}}{a}\left(\frac{C_{3} G m_{e} Z}{A_{0} m_{p}}+\frac{3 G}{5}\right)
\end{aligned}
$$

Minimizing this new expression for total energy, i.e., setting $d E_{t o t} / d a=0$ and $d^{2} E_{t o t} / d a^{2}>0$, the dependence on the stellar radius $a$ cancels out as before, leaving a limiting mass:

$$
M<M_{\text {limit }} \approx\left(\frac{5}{3} C_{1}\right)^{3 / 2} \frac{\left(\frac{\hbar c}{G}\right)^{3 / 2} Z^{2}}{\left(A_{0} m_{p}\right)^{2}}\left(1-\frac{10}{3} \frac{Z m_{e}}{A_{0} m_{p}}\right) .
$$

\subsubsection{Relativistic calculation}

Now using first order non-degenerate perturbation theory for the potential $U(r)=\left(2 \pi m_{e} G \rho r^{2} / 3\right)-2 \pi G \rho m_{e} a^{2}=$ $D_{1} r^{2}+D_{2}$, we have:

$$
\begin{aligned}
E_{n}^{1}= & \int \Psi^{\dagger} \gamma_{0} U(r) \Psi d^{3} r \\
= & A_{n}^{2} 4 \pi \int_{0}^{a}\left\{\left(D_{1} r^{4}+D_{2} r^{2}\right)\left|j_{0}\left(\frac{n \pi r}{a}\right)\right|^{2}\right. \\
& \left.\left.-\left(\frac{k}{\sqrt{k^{2}+\left(\frac{1}{\lambda_{e}}\right)^{2}}+\frac{1}{\lambda_{e}}}\right)^{2}\left|j_{1}\left(\frac{n \pi r}{a}\right)\right|^{2}\right)\right\} d r \\
= & D_{2}+\frac{a^{2} D_{1}}{6 \frac{1}{\lambda_{e}} n^{2} \pi^{2}}\left\{\frac{2}{\lambda_{e}}\left(3+n^{2} \pi^{2}\right)-9 \sqrt{\left(\frac{1}{\lambda_{e}}\right)^{2}+\frac{n^{2} \pi^{2}}{a^{2}}}\right\} .
\end{aligned}
$$

Now, to compute the correction to total kinetic energy of the completely degenerate electron gas :

$$
\begin{aligned}
T^{1}= & 2 \int_{0}^{n_{f}} E_{n}^{1} g(n) d n \\
= & \frac{\pi D_{2} n_{f}^{3}}{3}+\frac{\pi a^{2} D_{1}}{18 \frac{1}{\lambda_{e}} \pi^{2}}\left\{\frac{2}{\lambda_{e}} n_{f}\left(9+n_{f}^{2} \pi^{2}\right)\right. \\
& -\frac{27}{2 \pi}\left(n_{f} \pi \sqrt{\left(\frac{1}{\lambda_{e}}\right)^{2}+\frac{n_{f}^{2} \pi^{2}}{a^{2}}}\right. \\
& \left.\left.+a\left(\frac{1}{\lambda_{e}}\right)^{2} \log \left[\frac{n_{f} \pi+a \sqrt{\left(\frac{1}{\lambda_{e}}\right)^{2}+\frac{n_{f}^{2} \pi^{2}}{a^{2}}}}{a \sqrt{\left(\frac{1}{\lambda_{e}}\right)^{2}}}\right]\right)\right\}
\end{aligned}
$$


Now, $\frac{1}{\lambda_{e}}=\frac{m_{e} c}{\hbar} \approx 10^{12} m^{-1}$ is small compared to $n_{f} / a=$ $\left(\frac{3 N}{\pi}\right)^{1 / 3} / a \approx \frac{10^{20}}{7000 \times 10^{3}} \approx 10^{13} \mathrm{~m}^{-1}$ (taking radius a $\sim 7000$ $\mathrm{km})$, we can approximate Eq. (27) by,

$$
\begin{aligned}
T^{1} \simeq & \frac{\pi D_{2} n_{f}^{3}}{3} \\
& +\frac{\pi a^{2} D_{1}}{18 \frac{1}{\lambda_{e}} \pi^{2}} n_{f}^{3}\left[18 \frac{1}{\lambda_{e}} n_{f}^{-2}+2 \frac{1}{\lambda_{e}} \pi^{2}-\frac{27 \pi^{2}}{2 \pi a n_{f}}\right]
\end{aligned}
$$

For the leading order effect in the correction term, all powers of $\frac{1}{n_{f}}$ can be ignored to get:

$T^{1} \approx \frac{\pi D_{2} n_{f}^{3}}{3}+\frac{1}{9} \pi a^{2} D_{1} n_{f}^{3}$

Substituting the values of $D_{1}$ and $D_{2}$, we get:

$T^{1}=-\frac{4 G M^{2} m_{e} Z}{3 a A_{0} m_{p}}$

using $\rho=\frac{M}{\frac{4}{3} \pi a^{3}}$ and $N=Z \frac{M}{A_{0} m_{e}}$ where, $A_{0}$ is the average mass number of one nucleus of constituent particle of the star and $m_{p}$ is the mass of the proton, $\mathrm{N}$ is total number of nuclei multiplied by their charge number $\mathrm{Z}$ and the one with the subscript e is the mass of the electron. Thus the total energy of the star is:

$E=\frac{C_{1} \hbar c}{a}\left(\frac{Z M}{A_{0} m_{p}}\right)^{4 / 3}-\frac{3 G M^{2}}{5 a}-\frac{4 G M^{2} m_{e} Z}{3 a A_{0} m_{p}}$

The stability condition $\frac{d^{2} E}{d a^{2}}>0$ from Eq. (30) gives us the same order of magnitude correction term in the limiting mass as using the Schrodinger wave function.:

$$
\begin{aligned}
M & <M_{\text {limit }}=\left(\frac{5 C_{1}}{3}\right)^{3 / 2} \frac{\left(\frac{\hbar c}{G}\right)^{3 / 2} Z^{2}}{\left(A_{0} m_{p}\right)^{2}}\left[1+\frac{20 m_{e} Z}{9 A_{0} m_{p}}\right]^{-3 / 2} \\
& \approx\left(\frac{5 C_{1}}{3}\right)^{3 / 2} \frac{\left(\frac{\hbar c}{G}\right)^{3 / 2} Z^{2}}{\left(A_{0} m_{p}\right)^{2}}\left[1-\frac{10}{3} \frac{m_{e}}{A_{0} m_{p}} Z\right]
\end{aligned}
$$

The expression of the first two factors with first term in the last factor in parenthesis is the original limiting mass, while the second term is our leading correction arising from background-gravity of the star due to the nucleons, producing an effective gravitational potential inside the star. Clearly, this dimensionless correction term is a ratio of the mass of the electron to that of the proton, i.e., of the order of $10^{-4}$, and hence substantially smaller compared to the original contribution. This is as may have been expected, and in a sense justifies the neglect of the physical effect discussed here, in the incipient analysis. However, the effect is not so small as to be completely ignorable, especially if future observational studies require more precise results than what is available from the incipient analysis.

We also note en passant that the non-relativistic approximation indeed produces exactly the same correction to the limiting mass as the relativistic calculation, even though the ultra-relativistic nature of the electrons in the problem would prioritize the latter approach over the former.

\section{Conclusion and pending issues}

- The effect of a background gravitational potential on the electrons inside a White Dwarf is physical and has been demonstrated here to produce a change in the mass-limit of white dwarfs, the change being of the order of $10^{-4}$.

- One technical point is the simplifying assumption of restricting our estimation to the $s$-wave states of the wave functions. The reason for this restriction is primarily because what we are attempting to determine is an order-of-magnitude change of the limiting mass due to the effect of background gravity on the electron equation of state. This determination entails computing the first order change in the electron energy under the spherically symmetric perturbing background gravity potential, and then minimizing the total energy with respect to $a$. For the perturbation contribution to the energy to be small compared to unperturbed energy, the minimum value of $n$ is $n_{0} \simeq 10^{9}$. The largest possible value of $n$ corresponds to the Fermi level and is $O\left(10^{20}\right)$. Recalling that we are considering an estimate of the order-of-magnitude correction to the limiting mass, with $n \in\left[10^{9}, 10^{20}\right]$, it is unlikely that non-zero $l=O(1)$ states of the electrons in the spherically symmetric perturbing potential will have a major effect on the minimum total energy with first order perturbative corrections. Our restriction to $l=0$ states for the energy minimization, or determination of the density of states, leading to the limiting mass follows the same restriction followed in the incipient papers on the limiting mass [8-12], which is obvious from their use of the energy quantization corresponding to the momentum spectrum $p=\frac{n \pi \hbar}{a}$ in place of $p=\frac{\zeta_{n l} \pi \hbar}{a}$ where $\zeta_{n l}$ is the nth zero of the lth spherical bessel function of the first kind. The inclusion of $l \neq 0$ states, even for the unperturbed spherical well will no doubt alter the energy spectrum non-trivially, leading to a result that is no longer a simple analytic function of $n$. For large enough $l$, one may expect modifications to the limiting mass, as a result, whose determination lies outside the purview of the present work. The effect of such states on the first order perturbation correction to the limiting mass, due to the effective background gravity, is an interesting technical issue which we hope to resolve in a future publication [21].

- This change will also affect the absolute luminosity of Type-Ia Supernovae as calculated from the Mass-Limit. Since Type-Ia Supernovae act as Standard candles, our correction might have a significant effect in the measured value of the cosmological parameters. In light of 
the second point, considering type 1-a supernovae to be thermonuclear explosions of super chandrasekhar mass white dwarfs, the total energy released in such an explosion and hence the luminosity can be thought to be approximately proportional to the mass of its progenitor times the speed of light squared. E.g., the luminosity $L=\alpha M_{\text {limit }} c^{2}$, when our correction term is incorporated, becomes: $L^{*} \approx L(1-0.0001)$. This will change the measured value of the luminosity distance by:

$$
\begin{aligned}
d_{L}^{*} & =\sqrt{\frac{L^{*}}{4 \pi F l u x}} \approx d_{L}(1-0.0001)^{\frac{1}{2}} \\
& \approx d_{L}(1-0.00005)
\end{aligned}
$$

The resultant change is indeed small but the corresponding change in cosmological parameters might be significant enough, given the ever-increasing precision currently being achieved in measurement of these parameters. From this standpoint, there seems to be scope for further research in the area.

- Our paper clearly ignores many corrections to the limiting mass, including those due to electron exchange and correlations, electron-ion electrostatic interactions, electron screening, finite temperature effects and so on. Many of these effects produce corrections more significant than the background gravity corrections considered in this paper (see, e.g., [20]). However, the physical effect we are considering in this paper is novel, and herein lies its importance. If such background gravitational aspects are considered for other more compact objects like neutron stars, the final effect on the equation of state may be significantly different from what is obtained by ignoring such effects altogether.

- Similarly, for denser white dwarfs, one might wonder whether general relativity ought to be used. There is some discussion on this in [2], regarding the use of general relativity. We are grateful to the anonymous referee for pointing out our omission in the earlier version of the paper.

- As far as observations directly related to the mass limit is concerned, our knowledge is scanty, except for one reported observation which concludes that the data reveals a white dwarf about twice the limiting mass [18]. It has been shown [4-7] that rotation and magnetic fields indeed produce a heavier white dwarf. We thank an anonymous referee for bringing these references to our attention.

- There is an argument in the literature due to Glendenning [19], originally for neutron stars, which could be adapted to the present context to argue that the effect of the background gravity on the electron equation of state ought to be negligibly small. Essentially, this argument employs the exterior Schwarzschild metric to argue that its change over the radius of an atom is negligibly small. We are not persuaded that this argument captures the gravitational effect of the matter in the interior of a white dwarf sufficiently accurately, especially the large densities present there. Indeed, the spacetime metric deep inside a white dwarf (and of course a neutron star) is expected to be quite different from an exterior Schwarzschild metric which solves only the vacuum Einstein equation. Our calculation above, even in the Newtonian gravity context, clearly shows that the effect of background gravity on the electron equation of state is not as negligible as Glendenning's argument would imply.

Acknowledgements We acknowledge interesting and useful discussions with Amitava Banerjee, Ritaban Chatterjee and Suchetana Chatterjee. One of us (PM) acknowledges very interesting correspondence with Amitabha Sen. We are especially grateful to an anonymous referee for pointing out to us the precursors to Chandrasekhar's derivation of the maximum mass using relativistic degeneracy, namely the work of W. Anderson and E. Stoner, and for bringing Ref. [3] to our attention. Her/his constructive criticism of earlier versions of the paper has helped produce what we hope is an improved version of the paper.

Data Availability Statement This manuscript has no associated data or the data will not be deposited. [Authors' comment: The main reason is that there is no data over and above the calculations presented in the paper, which lead to an order of magnitude estimate for the correction to the limiting mass of white dwarf stars.]

Open Access This article is distributed under the terms of the Creative Commons Attribution 4.0 International License (http://creativecomm ons.org/licenses/by/4.0/), which permits unrestricted use, distribution, and reproduction in any medium, provided you give appropriate credit to the original author(s) and the source, provide a link to the Creative Commons license, and indicate if changes were made.

Funded by SCOAP ${ }^{3}$.

\section{References}

1. G.A. Carvalho, R.M. Marinho Jr, M. Malheiro, General relativistic effects in the structure of massive white dwarfs. arXiv: $1709.01635 \mathrm{v} 3$

2. S.L. Shapiro, S.A. Teukolsky, Black Holes, White Dwarfs and Neutron Stars (Wiley, Hoboken, 2004)

3. M. Nauenberg, Edmund stoner and the discovery of the maximum mass of white dwarfs. J. Hist. Astron. 36, 297 (2008)

4. D. Chatterjee, A.F. Fantina, N. Chamel, J. Novak, M. Oertel, On the maximum mass of magnetised white dwarfs. Mon. Not. R. Astron. Soc. 456, 95 (2017). arXiv: 1610.03987

5. P. Bera, D. Bhattacharya, Mass-radius relation of strongly magnetized white dwarfs: nearly independent of Landau quantization. Mon. Not. R. Astron. Soc. 456, 3375 (2016). arXiv: 1405.2282v2

6. B. Franzon, S. Schramm, Effects of strong magnetic fields and rotation on white dwarf structure. Phys. Rev. D 92, 083006 (2015). arXiv: $1507.05557 \mathrm{v} 2$

7. K.A. Boshkayev, J.A. Rueda1, B.A. Zhami, Rotating hot white dwarfs. Astrophys. J. 762, 117 (2015). arXiv:1512.00052

8. W. Anderson, Z. Phys. 56, 851 (1929)

9. E. Stoner, Philos. Mag. 60, 944 (1930) 
10. L.D. Landau, Phys. Z. Sowjetunion 1, 285 (1932)

11. S. Chandrasekhar, An Introduction to the Study of Stellar Structure (Dover Publications Inc., New York, 1958)

12. S. Chandrasekhar, On stars, their evolution and their stability. Nobel Prize Lecture (1984)

13. A.S. Eddington, The Internal Constitution of the Stars (Cambridge University Press, Cambridge, 1926)

14. R.H. Fowler, Dense matter. Mon. Not. R. Astron. Soc. 87, 114 (1926)

15. V. Alonso, S. De Vincenzo, L. Mondino, Eur. J. Phys. 18, 315 (1997)

16. J.J. Sakurai, Modern Quantum Mechanics, Chapter 1 (Pearson Education Inc., London, 2011)
17. L.D. Landau, E.M. Lifshitz, Statistical Physics Part 1, Chapter XI (Elsevier, Amsterdam, 1980)

18. D.A. Howell, Nat. Lett. 443, 208 (2006)

19. N.K. Glendenning, Special and General Relativity, Chapter 4, Section 4.4 (Springer, Berlin, 2007)

20. A.Y. Potekhin, G. Chabrier, Contrib. Plas. Phys. 50, 82 (2010)

21. P. Maity, A. Ray, in preparation 